CP, 2018, Vol.7 - No13, pp. 9/26 ISSN 2014-6752. Girona (Catalunya). Universitat de Girona. MARTíNEZ MARTíNEZ, P.J.: Representación de la vacuna VPH en los mensajes de Twitter. Recibido: 7/11/2017 - Aceptado: 12/3/2018

\title{
Representación de la vacuna del VPH en los mensajes de Twitter
}

\section{The HPV vaccine portrayal in Twitter messages}

\author{
Autor \\ Dr. Pedro Javier Martínez Martínez \\ Profesor de Biología del Departament d'Ensenyament de Catalunya \\ pedro.martinez.biologia@gmail.com \\ ORCID: http://orcid.org/0000-0002-4205-9663 \\ Investigador InCom-UAB \\ Institut de la Comunicació (InCom-UAB) \\ Telèfon: +34 935812907 incom.uab.cat
}

\section{Resumen}

En la actualidad, cada vez son más las personas que utilizan Internet, y concretamente las redes sociales, para buscar información sobre cuestiones de salud. Sin embargo, se sabe poco sobre cómo se difunde la información sobre la vacuna del VPH en las redes sociales. Este artículo presenta un análisis y una comparación del contenido de los tuits publicados en inglés y en español, relacionados con la vacunación contra el VPH y el cáncer cervical. En total se examinaron 1.707 tuits en inglés y 418 en español. La mayoría de los mensajes en inglés eran comentarios personales publicados por los usuarios con un enfoque negativo respecto a la vacunación contra el VPH. En cambio, la mayoría de los tuits publicados en español eran anuncios comerciales de la vacuna, mensajes de organizaciones de salud y comentarios personales de los usuarios, y tenían una postura positiva hacia la vacunación contra el VPH. En ambos idiomas, gran parte de los mensajes contenían algún link para dirigir a los usua-

\section{Abstract}

Currently, more and more people are using the Internet, and specifically social networks, to find information on health issues. However, only a few is known about how information about the HPV vaccine is disseminated on social networks. This article presents an analysis and a comparison of the content of the tweets published in English and in Spanish, related to vaccination against HPV and cervical cancer. In total, 1.707 tweets in English and 418 in Spanish were examined. Most messages in English were personal comments posted by users with a negative focus on HPV vaccination. In contrast, most of the tweets published in Spanish were commercial advertisements for the vaccine, messages from health organizations and personal comments from users, and they had a positive stance towards vaccination against HPV. In both languages, a large part of the messages contained a link to direct users to informative health web pages. The results suggest that researchers of the HPV vaccine 
rios hacia páginas web informativas de salud. Los resultados sugieren que los investigadores de la vacuna del VPH han de tener en cuenta este medio de comunicación debido a su amplio alcance y su inmediata capacidad de difusión.

Palabras claves: VPH; papiloma; vacuna; salud; Twitter; comunicación. must take into account this means of communication due to its wide scope and its immediate diffusion capacity.

Keywords: HPV; papilloma; vaccine; health; Twitter; communication.

\section{Introducción}

La infección por el virus del papiloma humano (VPH) se produce por contacto sexual y es una de las enfermedades de transmisión sexual más difundidas en el mundo, donde cerca del $70 \%$ de la población se contagia en algún momento de su vida (Baseman \& Koutsky, 2005; Kahn \& Burk, 2007). La mayoría de estas infecciones son subclínicas, no reconocidas, y benignas (Bruni, Díaz, Castellsagué, Ferrer, Bosch \& de Sanjosé, 2010; Syrjanen, 2009). Desde que se descubrió que el VPH es la causa directa del cáncer de cuello uterino, se ha desarrollado una vacuna eficaz contra el VPH, aceptada por las organizaciones de salud e incluida en la cartera de vacunas recomendadas para la comunidad (Brotherton, Fridman, May, Chappell, Saville \& Gertig, 2011; Gilmour, Kanda, Kusumi, Tanimoto, Kami \& Shibuya, 2013).Hasta hoy en día las estrategias de prevención del VPH mediante la vacunación, se han centrado principalmente contra el cáncer cervical en mujeres. La vacuna contra el VPH es muy eficaz para prevenir el cáncer cervicouterino, pero también para evitar otras lesiones relacionadas como las verrugas genitales, anales y de pene (Ali et al., 2013; CDC, 2012). Los resultados de esta eficacia han sido probados en numerosos países desarrollados gracias a las campañas de vacunación, además existen numerosas evidencias de la importancia que tienen los hombres en el desarrollo de estas enfermedades (Hartwig, Syrjanen, Dominak-Felden, Brotons \& Castellsaguè, 2012; Chan et al., 2009). A pesar de ello, existen algunas controversias en la sociedad en torno a la vacuna contra el VPH (Vamos, McDermontt \& Daley, 2008).

En contraste con el modelo tradicional de internet, en el que los usuarios ven pasivamente los contenidos de una página creada por otros, la Web 2.0, denota un conjunto de herramientas que permiten a los usuarios crear y compartir el contenido de una manera interactiva y social. En este aspecto, las redes sociales se han convertido en uno de los principales medios de comunicación, interviniendo en el campo de la salud y la medicina (Eysenbach, 2008; Chu, Zamora, Young, Kurup \& Macario, 2010). La sociedad busca información sobre salud por numerosas razones: preocupación acerca de sí mismos, su familia o sus amigos. Algunas búsquedas se deben simplemente al interés general, tal vez instigado por una noticia o alguna publicación científica reciente (Hawn, 2009). Estudios recientes indican que entre el 75-80\% de los usuarios de internet buscan información sobre la salud y que la encuentran útil (Kata, 2010). El VPH es un argumento de búsqueda muy frecuente en los diversos motores de búsqueda, como se ejemplifica en algunos estudios (Tozzi, Buonuomo, Ciofi degli Atti, Carloni, Meloni \& Gamba, 2010). Aunque la calidad y la formalidad de muchas páginas sobre vacunación es cuestionable, y el contenido puede incluir información errónea. Además, la calidad de las páginas web de salud varía según el idioma de estas (Irwin, Wali, Fernando \& Schleyer, 2007). 
Twitter se ha convertido en la plataforma microblogging más popular, esta permite a los usuarios enviar y leer mensajes de texto breves limitados a 140 caracteres (tuits). Los tuits son publicados automáticamente en el timeline del usuario, donde cualquier otro usuario puede mencionar, responder y retuitear el mensaje publicado, generando un debate público (Chaudhry, Glodé, Gillman \& Miller, 2012; Torrente, Martí \& Escarrabill, 2012). En la actualidad, hay un creciente interés en analizar datos obtenidos de los medios sociales, y de Twitter en particular, para obtener información acerca de las actividades en línea y su impacto (Prochaska, Pechmann, Kim \& Leonhardt, 2012; Kim, Seok, Oh, Lee \& Kim, 2013). El servicio cuenta con más de 330 millones de usuarios y procesa unos 500 millones de tuits por día (Statista, 2017). Algunos análisis de Twitter revelan que la mayor parte de los tuits contienen conversaciones entre los usuarios, solo de interés para los sujetos involucrados, enlaces a otras noticias, spam o propaganda (Signorini, Segre \& Polgreen, 2011). A pesar del alto nivel de ruido, Twitter contiene información muy útil, ya que es una plataforma que permite informar in situ y a tiempo real (Salathé \& Khandelwal, 2011), además tiene un número ilimitado de fuentes de información, permite incluir links en los mensajes, la información es inmediata, gracias a los smartphones se puede acceder en cualquier lugar y en cualquier momento (Torrente et al., 2012), e incluso se está estudiando la posibilidad de citar artículos científicos por esta vía (Sakaki, Okazaki \& Matsuo, 2010); todo esto está provocando un cambio en los patrones tradicionales de comunicación sobre la salud (Priem \& Costello, 2010).

Cada vez más, los hospitales e instituciones de salud están adoptando esta red social para la comercialización y la educación del paciente (Chaudhry et al., 2012), uno de los pioneros fue el Hospital Sant Joan de Déu (@HSJDBCN) (Torrente et al., 2012). De la misma manera, cada vez más son los pacientes que presentan sus problemas de salud a profesionales a través de Twitter en busca de consejos (Takhteyev, Gruzd \& Wellman, 2012). Se han hecho diversos estudios analizando esta red social en relación a temas de salud: tabaco (Prochaska et al., 2012), hábitos nutricionales (Sugawara et al., 2012), problemas dentales (Hingle et al., 2013), antibióticos (Heaivilin, Gerbert, Page \& Gibbs, 2011), virus de la gripe (Scanfeld, Scanfeld \& Larson, 2010), etc.; pero no hay ninguno que compare las impresiones obtenidas de los tuits en inglés y en español sobre la vacuna contra el VPH.

El propósito de este estudio es presentar un análisis del contenido de los mensajes de Twitter en inglés y en español, las dos lenguas más habladas en el mundo junto al chino y las más utilizadas en internet, acerca de la vacunación contra el VPH, en el que se exponga el tipo de fuente de emisión de la publicación, la posición de aceptabilidad hacia la vacuna, el grado de influencia del mensaje y su país de origen. Los resultados nos pueden dar pistas sobre las fuentes de información que hay que promover, los problemas que deben hacer frente los profesionales de la salud en un futuro inmediato, y nos pueden ayudar a escoger la dirección de las nuevas campañas de salud.

\section{Material y métodos}

Para analizar el contenido de los tuits acerca de la vacunación contra el VPH, se utilizó NodeXL como herramienta de análisis de Twitter, utilizada en otros estudios similares (Lulic \& Kovic, 2013). Se realizó una búsqueda de los tuits tanto en lengua inglesa como en castellano publicados entre el 1 de agosto de 2017 y el 31 de agosto de 2017 relacionados con la vacuna del VPH. Las palabras clave para la búsqueda de tuits en inglés fueron: "human papillomavirus vaccine", 
"HPV vaccine", "Gardasil vaccine" y "Cervarix vaccine", mientras que en español fueron: "vacuna virus papiloma humano", "vacuna VPH", "vacuna Gardasil" y "vacuna Cervarix". El análisis de los tuits se realizó inmediatamente después de la búsqueda, eliminándose de la muestra final los mensajes con alguno de los siguientes criterios de exclusión: tuits que estuvieran en un idioma distinto al inglés o al castellano; tuits duplicados, muchos de ellos son publicados por el mismo usuario pero en distintos días; y, los tuits irrelevantes, que no contenían información relacionada con Gardasil'. Cervarix , o vacunación contra el virus del papiloma humano (VPH), fueron eliminados.

Los tuits se categorizaron según el tipo de publicación en: noticias (locales y nacionales), anuncios, publicaciones realizadas por organizaciones de salud (entre las que destacan las de expertos y profesionales de la salud), preguntas de los usuarios, y comentarios de los mismos. Para determinar los mensajes más populares y socialmente influyentes, los tuits fueron clasificados según la acción con la que el emisor la difundió en: mención (influencia alta), respuesta (influencia media) y tuits (influencia baja). Además, se anotó el país de publicación de cada tuit, se calculó el número total de mensajes de las categorías anteriores, y se creó un Word Cloud, como en otros estudios similares (Hingle et al., 2013; Heaivilin et al., 2011), donde se representa de forma clara y visual el contenido de los tuits analizados. Y finalmente, se comparó cada una de las categorías analizadas entre los mensajes publicados en inglés y los publicados en español.

\section{Resultados}

Se encontraron un total de 3.431 tuits, de los cuales 2.316 eran inglés y 1.115 en español, utilizando las palabras clave. Entre estos, 2.122 fueron exclusivos tras aplicar los criterios de exclusión y fueron analizados, 1.704 en inglés y 418 en español. La mayoría ( $n=873 ; 51 \%)$ de los tuits en inglés, se obtuvieron mediante las palabras clave "HPV vaccine", se encontraron bastantes mensajes $(n=600 ; 35 \%)$ a partir de las palabras "Gardasil vaccine", unos pocos mensajes $(n=226 ; 13 \%)$ respondían a las palabras clave "Cervarix vaccine", mientras que solo se halló una pequeña cantidad de tuits mediante las palabras clave "human papillomavirus vaccine" ( $n=5 ; 1 \%)$. En español, la inmensa mayoría $(n=367 ; 87 \%)$ de los tuits, se obtuvieron mediante las palabras clave "vacuna VPH", se encontraron unos pocos mensajes ( $n=42 ; 10 \%)$ a partir de las palabras "vacuna Gardasil", mientras que solo se halló una pequeña cantidad de tuits mediante las palabras clave "vacuna virus papiloma humano" $(n=8 ; 2 \%)$ y "vacuna Cervarix" ( $n=1 ; 0.1 \%)$.

El número de tuits según la acción de publicación del texto fue el siguiente: mención ( $n=1.109$; $52 \%)$, de los cuales el $22 \%$ eran retuits; tuit ( $n=928 ; 44 \%)$, de los cuales el $12 \%$ eran retuits; $y$, respuesta ( $n=85 ; 4 \%$ ), de los cuales el $0.3 \%$ eran retuits; de los 2.122 tuits analizados, el $34 \%$ eran retuits. De forma Independiente, se obtuvo que los tuits en inglés siguieron el siguiente orden: mención ( $n=837 ; 49 \%$ ), de los cuales el $67 \%$ eran retuits; tuit ( $n=806 ; 47 \%)$, de los cuales el $33 \%$ eran retuits; y respuesta $(n=61 ; 4 \%)$, de los cuales el $0.5 \%$ eran retuits; globalmente el $35 \%(n=598)$ de los mensajes en inglés fueron retuiteados. $Y$, los tuits en español siguieron el siguiente orden: mención ( $n=272 ; 65 \%)$, de los cuales el $53 \%$ eran retuits; tuit $(n=122 ; 29 \%)$, de los cuales el $43 \%$ eran retuits; $y$, respuesta $(n=24 ; 6 \%)$, de los cuales el $3 \%$ eran retuits. Globalmente el 29\% $(n=120)$ de los mensajes fueron retuiteados (Gráfica 1). 


\section{Gráfica 1. Número de tuits en cada idioma según la acción de la publicación.}

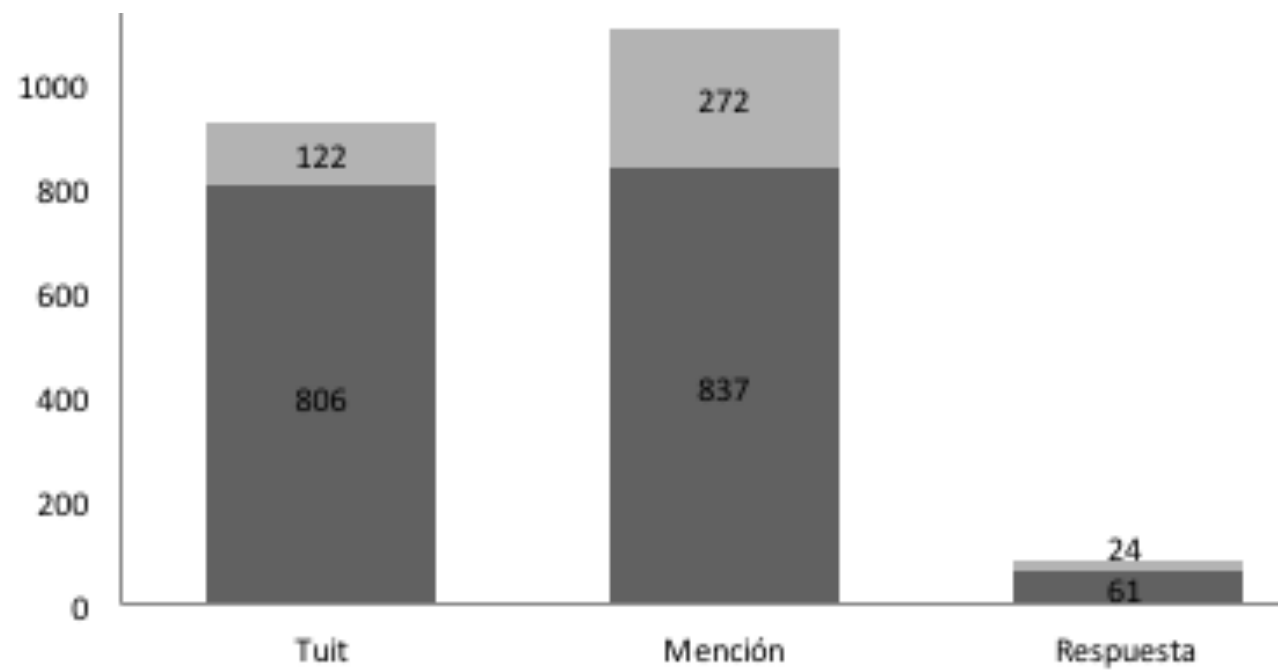

- Tuits en inglés = Tuits en espafiol

Con respecto a la postura adoptada por los tuits, de forma general, se encontró que la mayoría mantenían una posición negativa hacia la vacunación contra el VPH $(n=1.301 ; 61 \%)$ de los cuales el $23 \%$ eran retuits, mientras que una minoría $(n=597,28 \%)$ mantenía una posición positiva (8\% eran retuits). Además, se encontró una pequeña cantidad con una postura neutral hacia la vacuna $(n=224 ; 11 \%)$ siendo el $3 \%$ retuits. Analizando cada lengua de forma independiente, casi tres cuartas partes $(n=1.236,73 \%)$ de los tuits en inglés mostraron una postura negativa hacia la vacuna, de los cuales el $76 \%$ eran retuits; mientras que una minoría ( $n=289 ; 17 \%$ ) la representaban como algo positivo, de los cuales el $15 \%$ eran retuits; entre ellos se encontró una pequeña parte de mensajes $(n=179 ; 10 \%)$ que tenían una postura neutra hacia la vacunación contra el VPH, de los cuales el $9 \%$ eran retuits. En cambio, casi tres cuartas partes $(n=308$; $74 \%$ ) de los tuits analizados representaban la vacunación contra el VPH como algo positivo, de los cuales el $66 \%$ eran retuits; mientras que una minoría $(n=65 ; 16 \%)$ la representaban como algo negativo, de los cuales el $24 \%$ eran retuits; además se encontró una pequeña parte de mensajes $(n=45 ; 10 \%)$ que tenían una postura neutra hacia la vacunación contra el VPH, se los cuales el $10 \%$ eran retuits (Gráfica 2 ). 


\section{Gráfica 2. Número de tuits según la postura hacia la vacuna contra el VPH.}

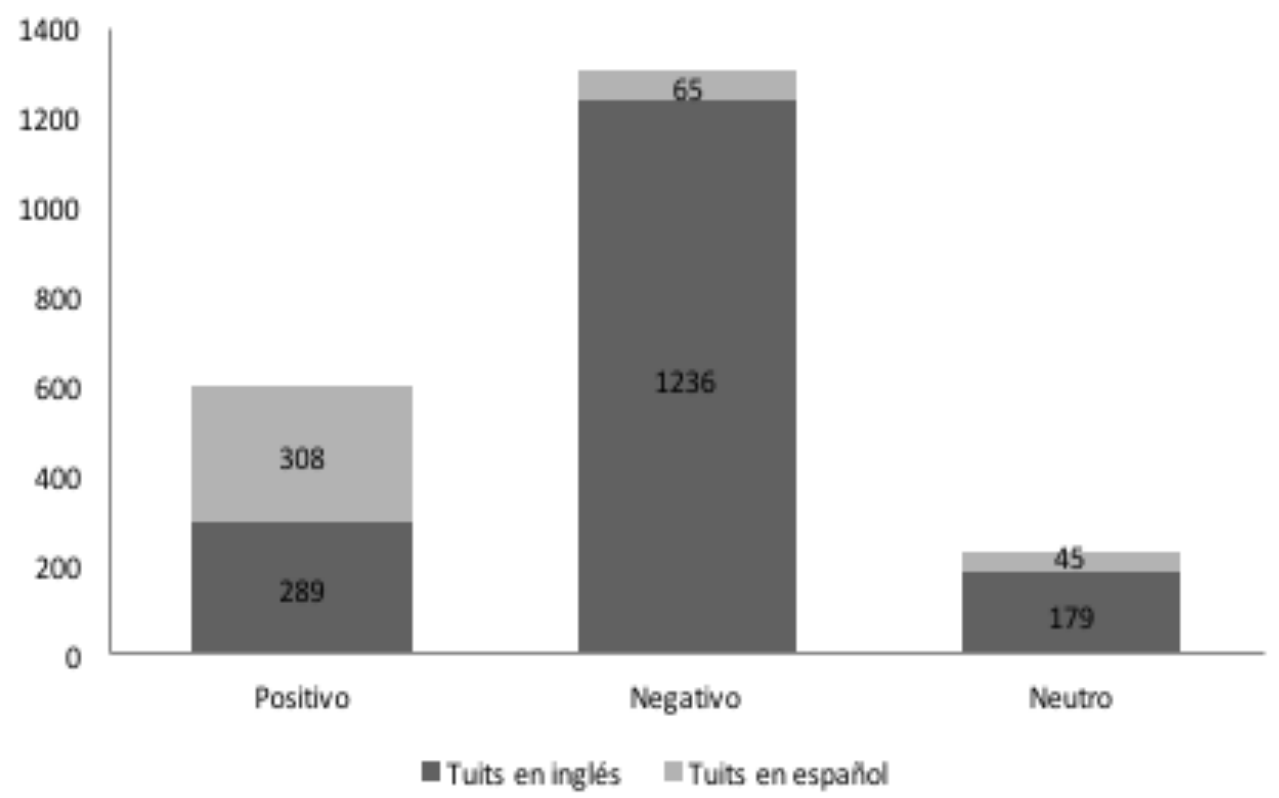

De forma global, el número de tuits según el tipo de publicación siguió el siguiente orden: comentarios personales de los usuarios de Twitter $(n=1.111 ; 53 \%)$, de los cuales el $21 \%$ eran retuits; noticias nacionales $(n=379 ; 18 \%)$, de los cuales el $3 \%$ eran retuits; publicaciones de organizaciones de salud ( $n=262 ; 12 \%)$, de los cuales el $4 \%$ eran retuits; anuncios $(n=195 ; 9 \%)$, de los cuales el $2 \%$ eran retuits; noticias locales ( $n=107 ; 5 \%)$, de los cuales el $2 \%$ eran retuits; $y$, preguntas de los usuarios a profesionales de la salud y a otros usuarios $(n=68 ; 3 \%)$, de los cuales el 1\% eran retuits. El número de tuits en inglés según el tipo de publicación siguió un orden similar: comentarios personales de los usuarios de Twitter ( $n=1.031 ; 59 \%)$, de los cuales el $70 \%$ eran retuits; noticias nacionales $(n=350 ; 21 \%)$, de los cuales el $11 \%$ eran retuits; publicaciones de organizaciones de salud ( $n=180 ; 11 \%)$, de los cuales el $10 \%$ eran retuits; preguntas de los usuarios a profesionales de la salud y a otros usuarios $(n=51 ; 3 \%)$, de los cuales el $2 \%$ eran retuits; noticias locales ( $n=49 ; 3 \%)$, de los cuales el 3\% eran retuits; $y$, anuncios $(n=43 ; 3 \%)$, de los cuales el $4 \%$ eran retuits. En español siguió en siguiente orden: anuncios ( $n=152 ; 36 \%)$, de los cuales el $15 \%$ eran retuits; publicaciones de organizaciones de salud ( $n=82 ; 20 \%)$, de los cuales el $27 \%$ eran retuits; comentarios de los usuarios de Twitter $(n=80 ; 19 \%)$, de los cuales el $20 \%$ eran retuits; noticias locales ( $n=58 ; 14 \%$ ), de los cuales el $23 \%$ eran retuits; noticias nacionales $(n=29 ; 7 \%)$, de los cuales el $9 \%$ eran retuits; preguntas de los usuarios a profesionales de la salud y a otros usuarios ( $n=17 ; 4 \%$ ), de los cuales el $5 \%$ eran retuits (Gráfica 3) (Tabla 1). 


\section{Gráfica 3. Número de tuits en cada idioma según el tipo de publicación}

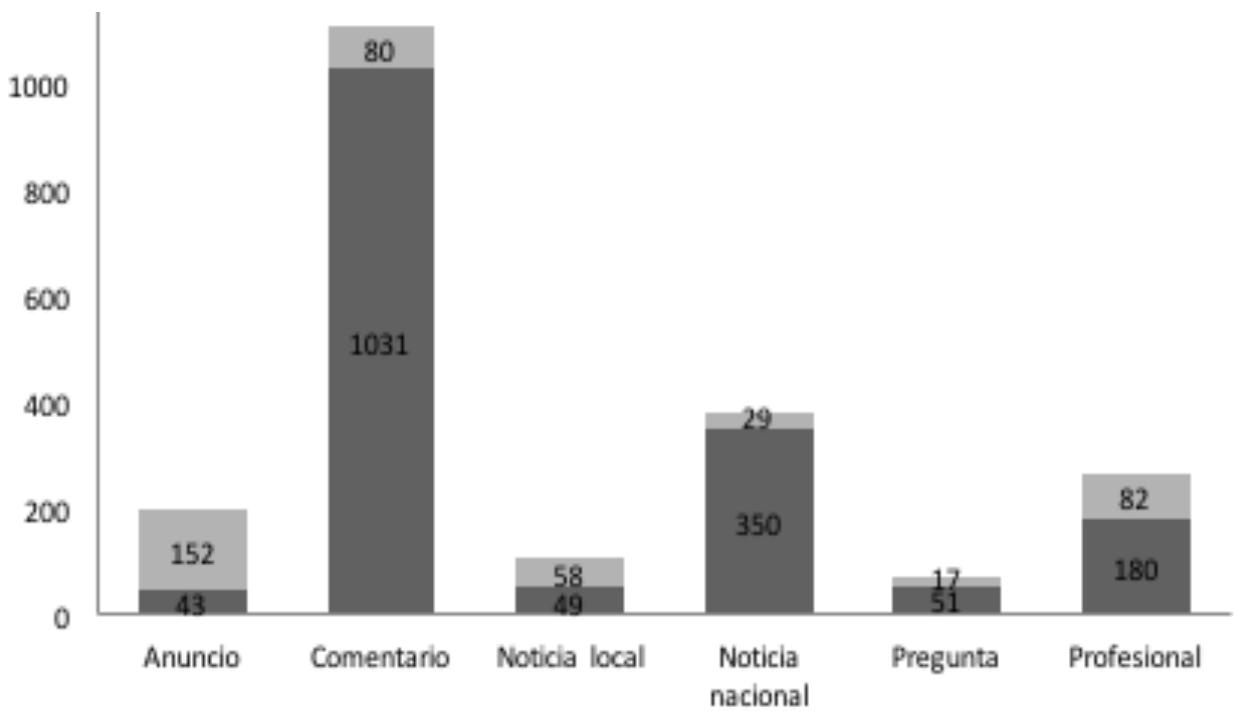

- Tuits en inglés = Tuits en español

Según el número de publicaciones por país podemos encontrar en primer lugar a Estados Unidos con un $54 \%(n=1.142)$ de las publicaciones, seguido por Venezuela con un $13 \%(n=267)$. Con algo menos de publicaciones le sigue Reino Unido $(n=124 ; 6 \%)$, Sudáfrica $(n=106: 5 \%)$ y Canadá ( $n=105 ; 5 \%)$ (Gráfica 4). 
Tabla 1. Tabla resumen de los datos obtenidos.

Según la acción de publicación:

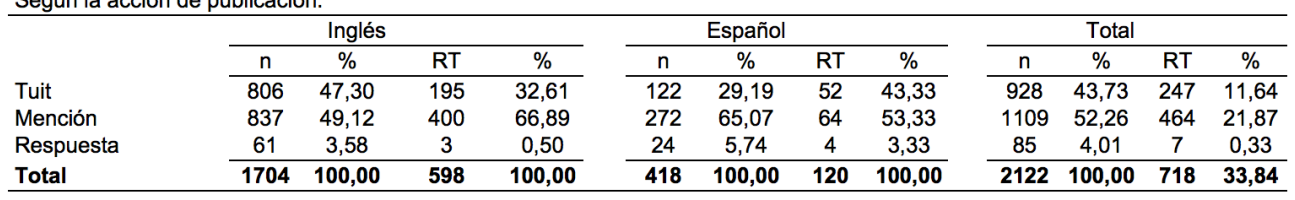

Según la posición hacia la vacuna contra el VPH:

\begin{tabular}{|c|c|c|c|c|c|c|c|c|c|c|c|c|}
\hline & \multicolumn{3}{|c|}{ Inglés } & & \multicolumn{4}{|c|}{ Español } & \multicolumn{4}{|c|}{ Total } \\
\hline & $\mathrm{n}$ & $\%$ & RT & $\%$ & $\mathrm{n}$ & $\%$ & RT & $\%$ & $\mathrm{n}$ & $\%$ & RT & $\%$ \\
\hline Positivo & 289 & 16,96 & 87 & 14,55 & 308 & 73,68 & 79 & 65,83 & 597 & 28,13 & 166 & 7,82 \\
\hline Negativo & 1236 & 72,54 & 457 & 76,42 & 65 & 15,55 & 29 & 24,17 & 1301 & 61,31 & 486 & 22,90 \\
\hline Neutro & 179 & 10,50 & 54 & 9,03 & 45 & 10,77 & 12 & 10,00 & 224 & 10,56 & 66 & 3,11 \\
\hline Total & 1704 & 100,00 & 598 & 100,00 & 418 & 100,00 & 120 & 100,00 & 2122 & 100,00 & 718 & 33,84 \\
\hline \multicolumn{13}{|l|}{ Según el tipo de p } \\
\hline & \multicolumn{3}{|c|}{ Inglés } & & \multicolumn{3}{|c|}{ Español } & & \multicolumn{3}{|c|}{ Total } & \\
\hline & $\mathrm{n}$ & $\%$ & RT & $\%$ & $\mathrm{n}$ & $\%$ & RT & $\%$ & $\mathrm{n}$ & $\%$ & RT & $\%$ \\
\hline Anuncio & 43 & 2,52 & 21 & 3,51 & 152 & 36,36 & 18 & 15,00 & 195 & 9,19 & 39 & 1,84 \\
\hline Comentario & 1031 & 60,50 & 420 & 70,23 & 80 & 19,14 & 24 & 20,00 & 1111 & 52,36 & 444 & 20,92 \\
\hline Noticia local & 49 & 2,88 & 20 & 3,34 & 58 & 13,88 & 28 & 23,33 & 107 & 5,04 & 48 & 2,26 \\
\hline Noticia nacional & 350 & 20,54 & 63 & 10,54 & 29 & 6,94 & 11 & 9,17 & 379 & 17,86 & 74 & 3,49 \\
\hline Pregunta & 51 & 2,99 & 12 & 2,01 & 17 & 4,07 & 6 & 5,00 & 68 & 3,20 & 18 & 0,85 \\
\hline Profesional & 180 & 10,56 & 62 & 10,37 & 82 & 19,62 & 33 & 27,50 & 262 & 12,35 & 95 & 4,48 \\
\hline Total & 1704 & 100,00 & 598 & 100,00 & 418 & 100,00 & 120 & 100,00 & 2122 & 100,00 & 718 & 33,84 \\
\hline
\end{tabular}

n: número de tuits; $\mathrm{RT}$ : retuits

\section{Gráfica 4. Número de tuilts publicados en inglés o en español en cada país.}

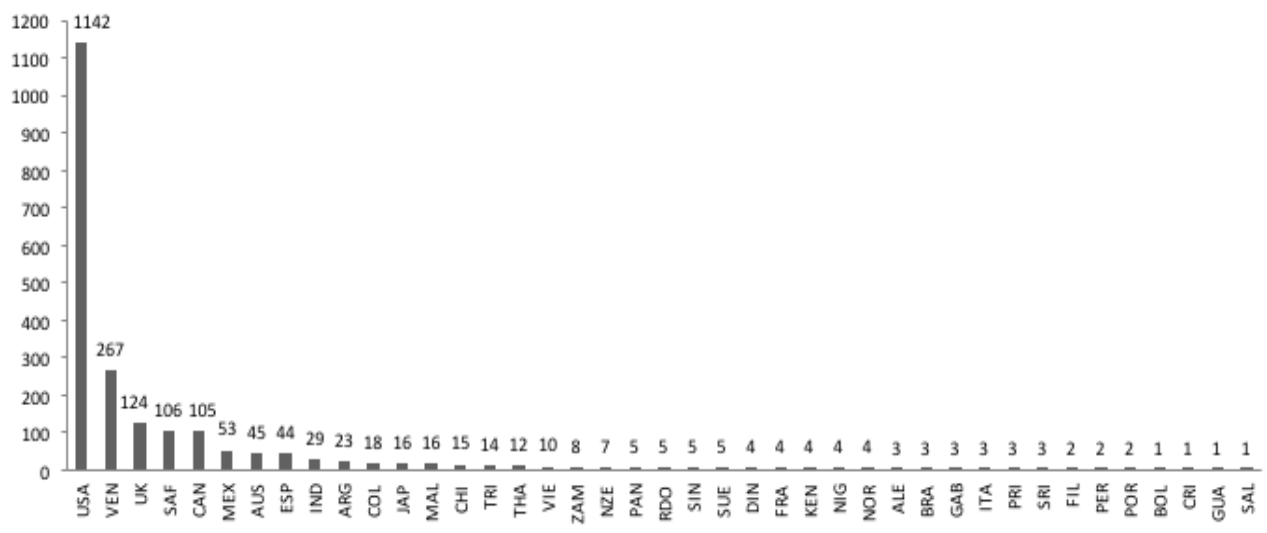

USA: Estados Unidos; VEN: Venezuela; UK: Reino Unido; SAF: Sudáfrica; CAN: Canadá; MEX: México; AUS: Australia; ESP: España; IND: India; ARG: Argentina; COL: Colombia; JAP: Japón; MAL: Malasia; CHI: China; TRI: Trinidad y Tobago; THA: Tailandia; VIE: Vietnam; ZAM: Zambia; NZE: Nueva Zelanda; PAN: Panamá; RDO: República Dominicana; SIN: Singapur; SUE: Suecia; DIN: Dinamarca; FRA: Francia; KEN: Kenia; NIG: Nigeria; NOR: Noruega; ALE: Alemania; BRA: Brasil; GAB: Gabón; ITA: Italia; PRI: Puerto Rico; SRI: SRI Lanka; FIL: Filipinas; y, PER: Perú; POR: Portugal; BOL: Bolivia; CRI: Costa Rica; GUA: Guatemala; y, SAL: El Salvador.

Finalmente se creó un Word Cloud, como en otros estudios similares, donde se representa de forma clara y visual el contenido de los tuits analizados, tanto en inglés como en español. Se 
representaron gráficamente las 150 palabras que aparecieron con más frecuencia de entre los 2.122 mensajes, correspondiendo las palabras de mayor tamaño a las que aparecieron con más frecuencia (Gráfica 5).

\section{Gráfica 5. Word Cloud de los tuits analizados en inglés y en español}

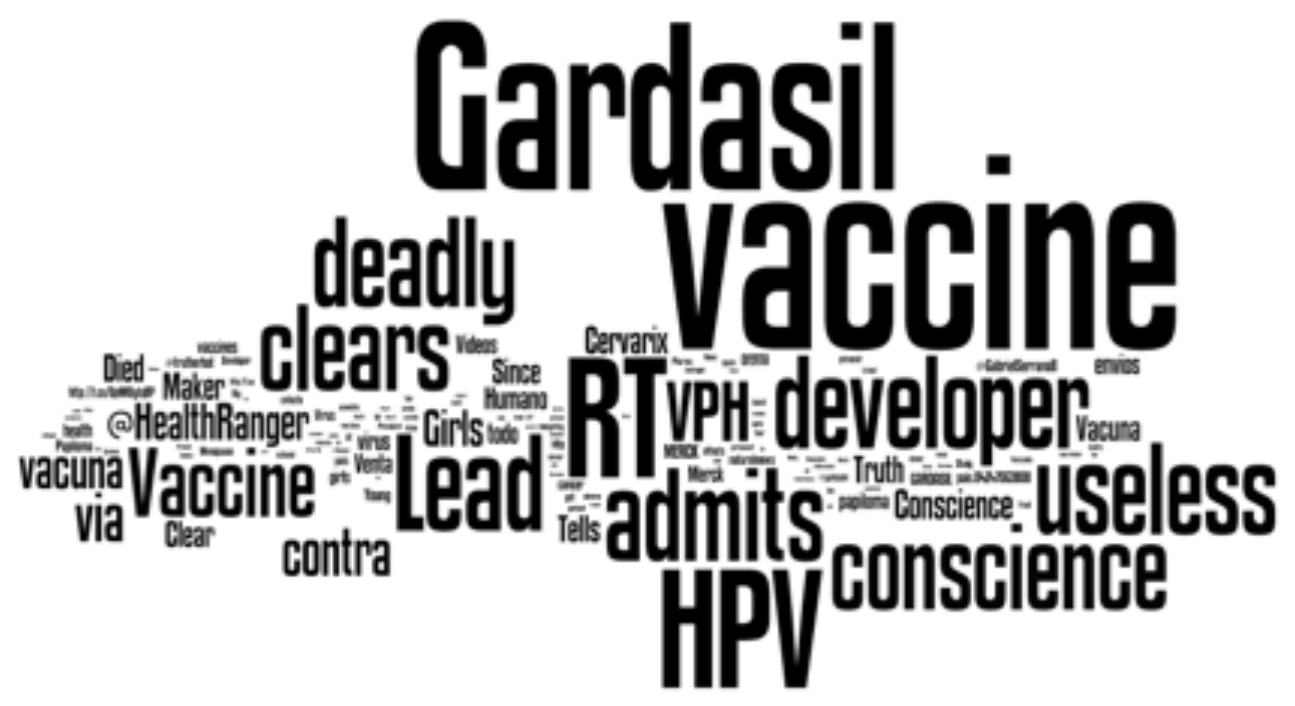

\section{Discusión}

Las vacunación es uno de los temas más controvertidos en el campo de la salud, por lo que se espera que la gente busque, cada vez más, información sobre la vacunación contra el VPH en la web, aunque se debe considerar que hay mucha desinformación e información negativa contra la vacuna del VPH (Kata, 2010; Bean, 2011). Los mensajes de Twitter se han convertido en una importante vía de información tanto para las organizaciones de la salud, como para las fuentes de noticias o para usuarios anónimos, para expresar opiniones a favor o en contra de la vacunación contra el VPH. A pesar de que aún no existe mucha investigación sobre Twitter en el campo de la salud, este estudio demuestra el dominio de la lengua inglesa en cuanto a número de tuits publicados sobre la vacunación contra el VPH, siendo Estados Unidos el país con más número de cuentas registradas.

Tal y como señalan algunas investigaciones, la calidad y rigurosidad de la información sobre el VPH disponible en internet puede variar con el idioma (Tozzi et al., 2010). El hallazgo más importante de este estudio fue que el $73 \%$ de los tuits en inglés publicados mantenían una posición negativa en relación a la vacunación contra el VPH, mientras que el $74 \%$ de los tuits publicados en español mantenían una posición positiva.

La mayoría del contenido de los tuits en inglés con una postura negativa hacia la vacuna, surgen de la difusión previa de noticias que ponen en duda su efectividad reportando diversos efectos adversos. Más de tres cuartas partes de los mensajes en ingles con una postura negativa eran retuits, lo que significa que sólo una cuarta parte fueron publicados exclusiva 
e independientemente por algún usuario. La gran mayoría de los mensajes en inglés eran comentarios de los usuarios, muchos de ellos con algún link que dirigía a otra página, y casi tres cuartas partes eran retuiteados. Además, la mayor parte de los mensajes eran menciones hacia otros usuarios. En este caso, se observa la enorme capacidad de difusión que tienen tanto los retuits como las menciones, consideradas en otras investigaciones como de alta influencia (Cha \& Gummadi, 2010). Entre los países de habla inglesa destaca el número de tuits procedentes de EEUU, foco del origen de las informaciones con una connotación negativa y su consecuente rechazo hacia la vacuna. Otros países angloparlantes en los que se encontraron bastantes tuits relacionados con la vacuna, pero con valores más discretos fueron: Sudáfrica, Canadá, Inglaterra y Australia.

En cambio, la mayoría de tuits publicados en español fueron anuncios de comerciales o distribuidores de la vacuna y anuncios de campañas de vacunación contra el VPH en centros de salud, sobre todo de Venezuela, y todos ellos con una postura positiva hacia la vacuna. Twitter es una plataforma en la que la información que se publica es inmediata y, normalmente, los sucesos publicados acaban de ocurrir en el momento de escribir el mensaje (Signorini et al., 2011), de esta manera se entiende el hecho de que en Venezuela, con un número menor de usuarios de Twitter que España y México, haya tanta cantidad de tuits en comparación con otros países donde la campaña de vacunación se realizó años atrás, como en España. Por el contrario, los comentarios en español de los usuarios anónimos de Twitter fueron, en gran medida, negativos e incluso se encontró una gran cantidad de comentarios con un contenido neutro hacia la vacuna. Estos comentarios, generalmente, relataban experiencias propias de los usuarios, experiencias de alguna persona cercana a ellos, mitos y creencias populares, por lo que se trataba de mensajes subjetivos o sin ninguna base científica. De forma similar a como ocurrió con los tuits en inglés, se pudo observar una gran cantidad de menciones y de retuits en torno a las publicaciones en castellano.

Los países de habla hispana son los que dominaron en cuanto a número de tuits publicados en castellano, destacando la elevada proporción de mensajes publicados en Venezuela, México, España, Argentina y Colombia. Sin embargo, hay que destacar el número de publicaciones en español realizadas desde Estados Unidos, concretamente en el sur del país, entre los países de habla no hispana. Los mensajes de los profesionales y organizaciones de la salud fueron los más numerosos, en proporción, entre las publicaciones en español. Es importante que los profesionales de la salud tengan un conocimiento básico de este tipo de servicio y la naturaleza de la información relacionada con la salud que se publica.

Tras la realización de este estudio, se ha podido observar que Twitter puede servir de herramienta para identificar la desinformación y errores conceptuales sobre la vacunación contra el VPH, así como para registrar movimientos antivacunales; además, dado su amplio poder de difusión, esta red social tiene un papel primordial en cuanto a la comunicación de la salud ya que puede intervenir en la aceptación social o el rechazo hacia de la vacuna.

La combinación de encuestas tradicionales junto a la información de la red social Twitter, puede ofrecer una representación diversa de diferentes grupos demográficos, y de esta manera ayudar a los profesionales de la salud a entender en qué lugares y por qué se rechaza una vacuna determinada. 


\section{Conclusión}

Los resultados de esta investigación demuestran que hay una amplia variedad de información en Twitter respecto a la vacunación contra el VPH y al cáncer cervical, predominando los tuits con gran capacidad de influencia o menciones, con una postura negativa hacia la vacunación contra el VPH en los tuits publicados en inglés, y positiva en los publicados en español. Un recurso tan mediático como Twitter tiene un gran potencial para influir tanto en las actitudes de los individuos como en la vacunación. Por ello, los médicos deben estar preparados para hacer frente a los pacientes con falsas impresiones, adquiridas a través de las noticias y los comentarios publicados por los usuarios de Twitter, acerca de los efectos adversos o la efectividad de la vacuna. En cambio, los investigadores y los profesionales de la salud tienen la oportunidad de emplear este recurso de información para la educación de los pacientes, haciendo frente a los mitos más comunes y transformándolos en ideas comprensibles, que aclaren las incertidumbres sobre la vacunación contra el VPH.

\section{Bibliografía}

- [1] Ali H, Donovan B, Wand H, Read TRH, Regan DG, Grulich AE, Fairley CK, Guy RJ. 2013. Genital warts in young Australians five years into national human papillomavirus vaccination programme: national surveillance data. BMJ, 346, 1-9.

- [2] Baseman JG, Koutsky LA. 2005. The epidemiology of human papilloma virus infections. J Clin Virol, 32(1), 16-24.

- [3] Bean SJ. 2011. Emerging and continuing trends in vaccine opposition website content. Vaccine, 29(10), 1874-1880.

- [4] Brotherton JM, Fridman M, May CL, Chappell G, Saville AM, Gertig DM. 2011. Early effect of the HPV vaccination programme on cervical abnormalities in Victoria, Australia: an ecological study. Lancet. 377, 2085-2092.

- [5] Bruni L, Díaz M, Castellsagué X, Ferrer E, Bosch FX, de Sanjosé S. 2010 Cervical human papillomavirus prevalence in 5 continents: meta-analysis of 1 million women with normal cytological findings. J Infect Dis, 202(12), 1789-1799.

- [6] Centers for Disease Control and Prevention (CDC). 2012. Morbidity and Mortality Weekly Reports (MMWR), 61(15), 258-261.

- [7] Cha M, Gummadi KP. 2010. Measuring User Influence in Twitter: The Million Follower Fallacy. ICWSM '10: Proceedings of international AAAI Conference on Weblogs and Social.

- [8] Chan S, Ng B, Lo W, Cheung T, Chung T. 2009. Adolescent girls' attitudes on human papillomavirus vaccination. J Pediatr Adolesc Gynecol, 22(2), 85-90.

- [9] Chaudhry A, Glodé LM, Gillman M, Miller RS. 2012. Trends in twitter use by physicians at the american society of clinical oncology annual meeting, 2010 and 2011. J Oncol Pract, 8(3), 173-178.

- $\quad$ [10] Chu LF, Zamora AK, Young CA, Kurup V \& Macario A. 2010. The role of social networking applications in the medical academic environment. Int Anesthesiol Clin, 48, 61-82.

- [11] Eysenbach G. 2008. Medicine 2.0: Social networking, collaboration, participation, apomediation, and openness. J Med Internet Res,10, 22.

- [12] Gilmour S, Kanda M, Kusumi E, Tanimoto T, Kami M, Shibuya K. 2013. HPV vaccination programme in Japan. Lancet, 382(9894), 768.

- [13] Greenberg L, D'Andrea G, Lorence D. 2004. Setting the public agenda for online health search: a white paper and action agenda. J Med Internet Res, 6(2), 18.

- [14] Hartwig S, Syrjanen S, Dominiak-Felden G, Brotons M, Castellsaguè X. 2012. Estima- 
tion of the epidemiological burden of human papillomavirus-related cancers and nonmalignant diseases in men in Europe: a review. BMC Cancer, 12, 30.

- [15] Hawn C. 2009. Take Two Aspirin And Tweet Me In The Morning: How Twitter, Facebook, And Other Social Media Are Reshaping Health Care. Health Aff, 28, 361-368.

- [16] Heaivilin N, Gerbert B, Page JE, Gibbs JL. 2011. Public health surveillance of dental pain via Twitter. J Dent Res, 90(9), 1047-1051.

- [17] Hingle M, Yoon D, Fowler J, Kobourov S, Schneider ML, Falk D, Burd R. 2013. Collection and visualization of dietary behavior and reasons for eating using Twitter. J Med Internet Res, 15(6), 125.

- [18] Irwin JY, Wali T, Fernando S, Schleyer T. 2007. Quality assessment of English and Spanish oral cancer websites. AMIA Annu Symp Proc. 11, 987.

- $\quad$ [19] Kahn JA, Burk RD. 2007. Papillomavirus vaccines in perspective. Lancet, 369, 2135-2137.

- [20] Kata A. 2010. A postmodern Pandora's box: anti-vaccination misinformation on the Internet. Vaccine, 28(7), 1709-1716.

- [21] Keelan J, Pavri-Garcia V, Tomlinson G, Wilson K. 2007. YouTube as a source of information on immunization: a content analysis. JAMA, 298(21), 2482-2484.

- [22] Kim E-K, Seok JH, Oh JS, Lee HW, Kim KH. 2013. Use of Hangeul Twitter to Track and Predict Human Influenza Infection. PLoS ONE, 8(7), 69305.

- [23] Lulic I, Kovic I. 2013. Analysis of emergency physicians' Twitter accounts. Med J, 30(5), 371-376.

- [24] Priem J, Costello KL. 2010. How and why scholars cite on twitter. Proceedings of the American Society for Information Science and Technology, 47, 1-4.

- [25] Prochaska JJ, Pechmann C, Kim R, Leonhardt JM. 2012. Twitter = quitter? An analysis of Twitter quit smoking social networks. Tobacco control, 21, 447-449.

- [26] Sakaki T, Okazaki M, Matsuo Y. 2010. Earthquake shakes Twitter users: realtime event detection by social sensors. Proceedings of the 19th international conference on World wide web. WWW '10. ACM New York, USA, 851-860.

- [27] Salathé M, Khandelwal S. 2011. Assessing vaccination sentiments with online social media: implications for infectious disease dynamics and control. PLoS Comput Biol, 7(10), 1002199.

- [28] Scanfeld D, Scanfeld V, Larson EL. 2010. Dissemination of health information through social networks: twitter and antibiotics. Am J Infect Control, 38(3), 182-188.

- [29] Signorini A, Segre AM, Polgreen PM. 2011. The Use of Twitter to Track Levels of Disease Activity and Public Concern in the U.S. during the Influenza A H1N1 Pandemic. PLoS ONE, 6(5), 19467.

- [30] Statista. Number of monthly active Twitter users worldwide from 1st quarter 2010 to 3rd quarter 2017 (in millions). Available from: https://www.statista.com/statistics/282087/ number-of-monthly-active-twitter-users/. Accessed November, 06, 2017.

- [31] Sugawara Y, Narimatsu H, Hozawa A, Shao L, Otani K, Fukao A. 2012. Cancer patients on Twitter: a novel patient community on social media. BMC Res Notes, 5, 699.

- [32] Syrjanen KJ. 2009. Annual disease burden due to human papillomavirus 16 and 18 infections in Finland. Scand J Infect Dis, 108, 2-32.

- [33] Takhteyev Y, Gruzd A, Wellman B. 2012. Geography of Twitter networks. Social Networks, 34, 73-81.

- [34] Torrente E, Martí T, Escarrabill J. 2012. A breath of Twitter. Rev Port Pneumol, 18(3), 137-141. 
- [35] Tozzi AE, Buonuomo PS, Ciofi degli Atti ML, Carloni E, Meloni M, Gamba F. 2010. Comparison of quality of internet pages on human papillomavirus immunization in Italian and in English. J Adolesc Health, 46(1), 83-89.

- [36] Vamos C, McDermott, Daley E. 2008. The HPV vaccine: Framing the arguments FOR and AGAINST mandatory vaccination of all middle school girls. J Sch Health, 78(6), 302309. 
